\title{
Bruit et vibrations des pompes
}

\author{
Alain Verry \\ EDF-DER
}

Sources de bruit et d'ondes de pression qui se propagent dans les tuyauteries, les pompes peuvent causer des dommages dans les circuits. Des efforts de recherche ont donc été faits pour améliorer leur comportement dynamique. Cette communication a été présentée par M. Alain Verry en clôture de la première conférence internationale organisée par la SHF du 7 au 9 juillet 1993 à Clamart sur le thème "Bruit et vibrations des pompes ». L'auteur donne une vue d'ensemble de la manifestation.

"Pump-generated noise and pressure pulsations are a cause of damage in the circuits. So efforts are being made to improve the dynamic performances of pumps. This paper was read by $M$. Alain Verry as the closing session of the first international conference which the Société hydrotechnique de France organized on the subject "Pump noise and Vibrations". The author gives an overview of the event.

\section{Un premier congrès}

Depuis une dizaine d'années, la conjonction d'intérêts industriels multiples a impulsé en France, un effort de recherche important visant à une meilleure maîtrise du bruit hydraulique des pompes et des vibrations induites dans les circuits associés. Selon qu'il s'agisse du secteur de la défense, de l'énergie, de l'aérospatial ou des applications domestiques, ce sont des préoccupations de discrétion acoustique, de fiabilité des installations ou de réduction de l'impact sur l'environnement qui prévalent.

Dans le cadre fédérateur du groupe de travail « Hydraulique et bruit " de la SOCIETE HYDROTECHNIQUE DE FRANCE. est apparue la conviction que les acquis étaient suffisants pour envisager l'organisation d'une conférence internationale qui permette de dresser un état de l'art et de favoriser le rapprochement des constructeurs de pompes, des concepteurs de circuits et des laboratoires de recherche

$\mathrm{Ce}$ projet a rapidement reçu le soutien de plusieurs sociétés savantes nationales et internationales telles l'AIRH et l'ASME, ainsi que l'appui d'EUROPUMP, de la Marine Nationale, de Sulzer-Pompes France et du CETIM. Fortement impliquée dans ce domaine, la Direction des Etudes et Recherches d'EDF a accueilli les cent quatre-vingts participants, venus de plus de quinze pays, dans son centre de recherche de Clamart, près de Paris.

\section{Les écoulements internes et la génération de bruit hydraulique}

Les pompes sont le siège d'écoulements rendus fortement instationnaires par la rotation de leur impulseur et par un niveau de turbulence très élevé à certain régimes. Les fluctuations de pression locales qui en résultent donnent naissance à des ondes de pression, appelées bruit hydrau- lique, qui se propagent dans la pompe et dans les réseaux de tuyauteries. Faiblement atténuées dans le fluide, ces fluctuations de pression cohérentes provoquent des vibrations de tuyauteries qui sont parfois responsables d'un endommagement sévère et généralement d'un niveau de bruit aérien beaucoup plus fort que celui généré par la volute de la pompe elle-même.

La compréhension des mécanismes de génération de bruit hydraulique est une étape essentielle tant en ce qui concerne sa réduction à la source que la prévision du comportement acoustique et mécanique des installations. Au cours de sa conférence plénière, le Professeur BLAKE du David Taylor Naval Research Center a passé en revue les mécanismes de génération dominant selon le type de machine ou le régime de fonctionnement. M. JAREMCZAK et G. CAIGNAERT ont développé. sur la base des travaux de Simpson el Clarke, une méthode qui a fourni convenablement le bruit hydraulique de trois pompes conçues dans le cadre d'un groupe de travail de la SHF.

Comme l'ont montré plusieurs communications (J. Katz et al., M. Pluviose el J. Tourret, J.P. BArrand et al...), les progrès réalisés dans l'analyse expérimentale des champs de vitesse et de pression instationnaires locaux ainsi que dans le domaine de la modélisation numérique permettent d'espérer une avancée significative à assez court terme. Dans le cas des pompes, les modèles hydrodynamiques instationnaires tels que ceux développés au CREMHyG, à Grenoble (fig. l), ne sont pas encore couplés à des modèles de propagation acoustique. Ils ne permettent donc pas une prévision de la source équivalente. Ces modèles numériques devraient toutefois apporter une aide indispensable à l'interprétation des énormes volumes de mesures locales désormais disponibles dans plusieurs laboratoires. Une des difficultés de la modé- 


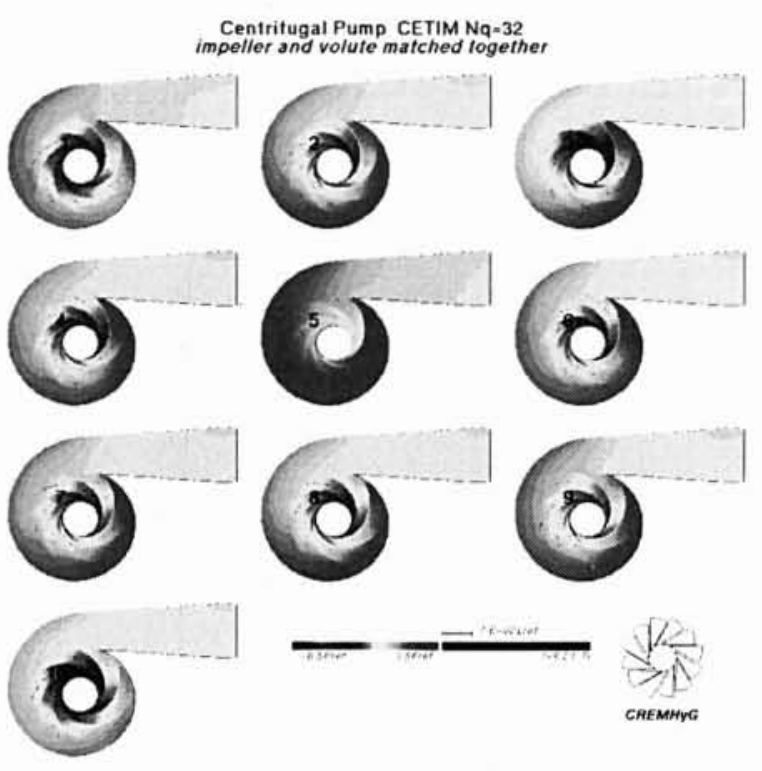

1. Champs de pression calculés dans une pompe au cours d'un cycle de rotation.

lisation sera la limite de validité des modèles de turbulence de type k-epsilon en écoulement instationnaire.

Ainsi, les analyses physiques et les modèles numériques ne fournissent pas encore de prévision précise des sources, ils donnent cependant des orientations pour la réduction du bruit à la source et la transposition au cas des machines réelles des sources mesurées sur modèle réduit car les lois de similitude à appliquer dépendent de la nature de la source (monopolaire pour le défilement des aubes des pompes centrifuges, dipolaire pour le défilement des aubes des pompes hélices, quadripolaire pour la turbulence).

\section{La conception de pompes plus silencieuses}

Les constructeurs ont commencé depuis plusieurs années à exploiter la connaissance des mécanismes de génération pour améliorer la conception hydroacoustique de leurs machines.

La première voie est naturellement la réduction des interactions entre la roue et le bec de volute ou le diffuseur. Pour ce faire, de nombreuses solutions ont été évoquées : augmentation de l'entrefer, réduction de la charge hydrodynamique sur les aubes en particulier au voisinage du bord de fuite, inclinaison des aubes... Ces modifications géométriques peuvent être très bénéfiques pour la réduction de l'effet du passage des aubes mais pénalisent rapidement le coût et les performances des machines industrielles. Une des difficultés majeures est l'obtention, malgré de grands entrefers, des courbes caractéristiques continûment descendantes imposées dans de nombreuses applications.

Les fonctionnements à débit réduit sont responsables d'une élévation du niveau de bruit hydraulique à la fréquence de passage des aubes. Mais surtout, l'apparition de recirculations fortement turbulentes à l'entrée de la roue et dans l'entrefer roue-diffuseur provoquent un accroissement important du spectre d'excitation à basse fréquence (typiquement de 0 à quelques dizaines de Hertz). Ce bruit hydraulique à basse fréquence est une source privilégiée d'excitation des tuyauteries et de la pompe elle-même. Bien que la compréhension de ces fonctionnements ait beaucoup progressé durant ces dix dernières années, leur maitrise reste l'un des objectifs importants des constructeurs. Outre une meilleure optimisation des œillards, les dispositifs « recirculateurs " installés dans la pièce d'aspiration constituent une technique prometteuse en particulier pour les roues à grand diamètre d'œillard et les inducteurs.

La cavitation est une autre source de fluctuations de pression. Le bruit créé à très haute fréquence n'est en général pas gênant pour les applications industrielles. Comme l'a montré U. BOLLETER, une légère cavitation ne provoque qu'un faible accroissement du bruit hydraulique en moyenne et basse fréquence. En revanche, elle modifie la matrice de transfert de la pompe, provoquant un découplage hydroacoustique partiel des tuyauteries amont et aval. Si les poches de cavitation sont plus développées, elles peuvent jouer un rôle de résonateur et conduire à des résonances marquées de la ligne d'aspiration à basse fréquence. Les poches peuvent également pulser sous l'effet d'une alimentation non axisymétrique de la roue. Une forte cavitation devient une source de bruit hydraulique importante mais elle doit en général être évitée en raison des risques d'érosion. Il est donc clair qu'une bonne maîtrise de la cavitation, grâce à une conception et une installation adéquate de la machine, est requise pour éviter des problèmes hydroacoustiques et vibratoires ensuite très difficiles à résoudre en exploitation.

Les matrices de transfert des pompes comportent des termes dont les variations, en fonction de la fréquence, sont significatives. Certains constructeurs (Sulzer / E. Klaui) ont engagé des recherches visant à tirer parti de ces variations, le cas échéant en tentant d'agir sur ces termes pour réduire le niveau de bruit produit par leurs machines. Pour les pompes de forte puissance spécifique, cette voie devra certainement être approfondie. L'étape suivante sera peut-être le contrôle actif !

La dynamique du rotor de la pompe n'est pas sans conséquence sur le comportement hydroacoustique. Les mouvements radiaux du rotor par rapport au stator ainsi que ses défauts de symétrie constituent une source de bruit hydraulique parfois significative. Réciproquement, les fluctuations de pression présentes dans le circuit de refoulement d'une pompe à volute peuvent exciter une fréquence propre de la ligne d'arbre. Cela a été montré sur un exemple rencontré récemment à EDF. Il faut également mentionner les instabilités parfois provoquées par les dispositifs d'équilibrage axiaux ou radiaux du rotor sous l'effet de pulsations de pressions des circuits d'aspiration ou de refoulement.

Enfin, les analyses menées à l'aide des méthodes d'intensimétrie hydroacoustique ont clairement confirmé le lien entre le comportement hydroacoustique de l'ensemble pompe-circuit et les efforts transmis au supportage de la pompe. La conception du dispositif de supportage doit en tenir compte. De plus, la position et l'orientation des 
tubulures d'aspiration et de refoulement conditionnent le comportement vibratoire de la machine. A titre d'exemple, les pompes verticales à volute qui ne sont pas supportées de façon très rigide ont souvent un niveau vibratoire important à la fréquence de passage des aubes de la roue.

\section{L'analyse des interactions machine-circuit}

La conférence donnée par le Dr BOLLETER constitue une excellente vue d'ensemble des mécanismes d'interaction hydroacoustique entre une pompe et son circuit. L'étude de ces interactions s'appuie sur des modèles linéarisés de propagation en ondes planes, traités le plus souvent grâce au formalisme des matrices de transfert. Ces modèles permettent de décrire aisément le comportement des réseaux de tuyauteries. La représentation des pompes pose plus de difficultés. Les coefficients de leur matrice de transfert et les sources associées ne peuvent être reliés simplement à leur géométrie et aux caractéristiques de l'écoulement. Plusieurs auteurs (Bolleter, LauroTrollat, de Jong, Whitson, TAVernier et al., JACOBPRENAT) ont proposé des modèles simplifiés de pompe pertinents pour leur application et permettant de limiter le nombre de paramètres à estimer ou à identifier expérimentalement. Des validations partielles de ces modèles ont été réalisées, mais il serait utile de les comparer et d'en déterminer les limites (type de pompe, bande de fréquence, régime de fonctionnement...).

Quel que soit le modèle utilisé pour la pompe, il reste encore aujourd'hui nécessaire de recourir à une identification expérimentale de ses paramètres pour obtenir une prévision précise du comportement hydroacoustique de l'installation. Dans ce domaine, plusieurs méthodes multicapteurs ont été exposées. Ces méthodes, basées également sur le modèle linéarisé de propagation en ondes planes fournissent aussi la célérité du son dans le tronçon de tuyauterie considéré. Les résultats obtenus sont généralement comparables. Il faut souligner la méthode établie par la Marine Nationale (instructions techniques présentées par M. Silhouette). Compte tenu du mode particulier d'installation des machines sur les sous-marins, la mesure d'un nombre très limité de paramètres est suffisante pour les caractériser. Dans le cas général, il est nécessaire d'utiliser des dispositifs d'excitation du circuit d'essai. Plusieurs de ces dispositifs ont été présentés. Celui qui est utilisé par l'EPFL et EDF est particulièrement efficace.

A moyen terme, la création de bases de données issues d'expériences de qualité devrait permettre d'améliorer la précision des études réalisées au stade de la conception. En tout état de cause, les constructeurs qui veulent optimiser sur le plan hydroacoustique leurs machines doivent maîtriser ces méthodes de caractérisation.

Au delả des méthodes purement hydroacoustiques qui permettent de diagnostiquer et de traiter les problèmes de résonance hydroacoustique, l'analyse du comportement vibratoire de l'installation nécessite la résolution du problème acoustique et vibratoire couplé. Il s'agit de prendre en compte les échanges d'énergie entre les modes hydroacoustiques et les modes vibratoires des tuyauteries modélisées comme des poutres. Ces échanges se produisent dans les coudes, les variations de section des tuyauteries ainsi que dans les pompes ou les vannes. Cette approche est mise en œuvre dans certains codes de calculs récents tels que CIRCUS développé à EDF. Ce code permet également d'introduire dans la simulation numérique des grandeurs (pression, débit, contrainte, déplacement) issues d'essais pour compenser la méconnaissance de certaines conditions aux limites ou de certains paramètres.

\section{Plusieurs cas d'application industrielle}

A l'évidence, les méthodes d'analyse acoustique et vibratoire des circuits n'ont pas encore atteint leur phase de maturité. Cependant, plusieurs auteurs ont montré l'intérêt de la mise en cuvre de ces méthodes sur des cas industriels concrets. Le premier domaine d'application est la résolution de problèmes rencontrés sur des circuits existants.

Grâce à une instrumentation sophistiquée et une modélisation hyroacoustique, ONTARIO HYDRO a pu expliquer l'origine de l'endommagement des assemblages combustibles de la centrale nucléaire de Darlington au Canada. L'excitation des assemblages a été fortement réduite par le changement du nombre d'aubes de la roue et de l'entrefer des pompes primaires.

Une étude vibroacoustique complète a permis à EDF d'expliquer la rupture d'un piquage situé sur un circuit de centrale nucléaire. Le modèle numérique qui a fourni une estimation du niveau de fluctuation des contraintes locales a été utilisé ensuite pour valider les modifications apportées au supportage et évaluer le gain en terme de durée de vie.

Pour éviter les interventions a posteriori, particulièrement coûteuses sur certains circuits tels que les circuits classés des centrales nucléaires ou pour satisfaire à des exigences particulières de discrétion acoustique, il sera certainement nécessaire de mener ces études dès la conception des installations. Il est déjà possible de prévoir les principaux modes hydroacoustiques et vibratoires, de déterminer les risques d'excitation par des fréquences discrètes et de localiser les zones les plus sollicitées du circuit. Ce type d'approche a été utilisée par Sulzer pour optimiser des circuits d'eau alimentaire de chaudière et par Westinghouse (E. SCHWIRIAN) pour analyser un circuit primaire de réacteur nucléaire. Le découplage machinecircuit retenu par la Marine Nationale est très efficace mais malheureusement ne peut être employé sur la plupart des circuits industriels pour des raisons de coût, de taille des tuyauteries ou de température du fluide pompé.

Dans le domaine des petites machines industrielles courantes, qui représentent une part très importante du chiffre d'affaires de l'industrie des pompes, il n'est naturellement pas question d'envisager des études aussi détaillées. Toutefois, la connaissance des mécanismes de génération de bruit hydraulique et de transfert vers le circuit est essentielle pour améliorer intrinsèquement les machines ainsi que pour codifier les méthodes de caractérisation et les conditions d'installation. La communication de MM. BRUNET et al. illustre cette démarche. Elle devrait permettre aux constructeurs de mieux répondre à l'attente expri- 
mée par leurs clients dans l'enquête menée par l'Association Française des Constructeurs de Pompes et présentée par M. DUMONT.

\section{Un bilan positif}

Comme l'a montré cette revue, naturellement non exhaustive, des communications présentées, ce premier congrès international sur le bruit et les vibrations des pompes a atteint son but. Les actes, qui peuvent être fournis sur demande par la SHF, constituent un état de l'art utile. La richesse des discussions indique que des contacts fructueux auront sûrement été noués. L'intérêt des participants pour ce domaine en pleine évolution nous incitera certainement à envisager dans quelques années une nouvelle manifestation sur le même thème.

\section{Table des matières / Table of contents}

\section{Conférence plénière / Key lecture}

Interactions dynamiques entre la pompe et le réseau de tuyauteries Interaction of pumps and piping systems with regard to pressure pulsations

Ulrich BOLLETER

\section{Thème 1 / Topic 1}

Analyse expérimentale des ecoulements instationnaires dans les pompes

Experimental analysis of unsteady flow in pumps

Effet du bec de volute sur l'écoulement et le bruit associé dans la volute d'une pompe centrifuge

The effect of blade-tongue interactions on the flow structure, pressure fluctuations and noise within a centrifugal pump

S. CHU, R. DONG, J.KATZ

Génération et propagation des fluctuations de pression dans une roue de pompe

Pressure fluctuations and waves within a pump impeller

J.P. BARRAND, M. JAREMCZAK, M. THALA

Etude du fonctionnement d'une pompe par visualisation du champ de pression non stationnaire

Analysis of the behaviour of a pump by visualization of the unsteady pressure field at partial flow

M. PLUVIOSE, F. LEZE, M. BESOMBES, J. TOURRET

Etude de l'écoulement instationnaire dans une pompe centrifuge fonctionnant à débit partiel

Analysis of unsteady flow in a centrifugal pump operating at partial flow rate

\section{F. HUREAU, J. KERMAREC, D. FOUCHER}

Ecoulement en entrée de rotors centrifuges de pompes et de compresseurs lors de fonctionnement à débit partiel

Flow similarities at inlet to centrifugal pumps and compressors during part load operation

\section{A. ENGEDA, N. WHITBECK}

Analyse des fluctuations de pression à l'aspiration d'une pompe fonctionnant à débit partiel

Experimental and theoretical study of the flow in the suction pipe of a centrifugal pump operating at partial flow rates

G. HUELSZ, C. SANTAOLARIA, E. RAMOS

Etude expérimentale du déclenchement d'instabilités dans une pompe à diffuseur lisse

Experimental analysis of Instabilities in a centrifugal pump with a vaneless diffusor

D. LEDUCQ

Analyse expérimentale des fluctuations liées à la cavitation dans l'inducteur d'une turbopompe
Experimental analysis of instabilities related to cavitation in an inducer of turbopump

J. de BERNARDI, F. JOUSSELLIN, A. VON KAENEL

\section{Thème 2 / Topic 2}

Calcul des écoulements instationnaires dans les pompes

Numerical analysis of unsteady flow in pumps

Analyse théorique et expérimentale du bruit hydraulique de 3 pompes centrifuges

Theoretical and experimental analysis of hydraulic noise on 3 centrifugal pump

\section{JAREMCZAK, G. CAIGNAERT}

Analyse numérique et expérimentale des écoulements instationnaires dans une pompe centrifuge. Interaction roue-volute

Numerical and experimental unsteady flow analysis in centrifugal pumps. Impeller and volute interaction

\section{CROBA, J.L. KUENY, F. HUREAU, J. KERMAREC}

Influence des tolérances géométriques de l'aubage sur les forces hydrodynamiques radiales instationnaires s'exerçant sur une roue centrifuge

Influence of geometrical blade tolerances on the unsteady radial hydrodynamic forces acting on centrifugal pump impellers

\section{G. VICTOROV, T. FENKO}

\section{Thème 3 / Topic 3}

Modélisation et prévision des sources de bruits hydrauliques dans les pompes et lois de similitude Modelling and prediction of fluid-borne noise and similarity laws

Les différentes sources de bruit hydrodynamiques générées par les aubages de pompe

Flow noise sources from blade system

V.M. PASHIN, E.L. MYSHINSKY, N.I. DUAN

Analyse expérimentale de la génération du bruit dans une pompe centrifuge

Experimental analysis of noise generation mechanism in a centrifugal pump

G. CHATEL, J. F. LAURO, C. TROLLAT 


\section{BRUIT ET VIBRATIONS DES POMPES}

Modélisation hydro-acoustique des pompes centrifuges Hydro-acoustical modelling for centrifugal pumps

\section{J. F. LAURO, C. TROLLAT}

Lois de similitude pour la prévision des efforts instationnaires et du bruit généré par les aubages

Scaling laws for prediction of unsteady forces and noise of rotating machinery

\section{Y. LEVKOVSKY}

Utilisation des paramètres énergétiques principaux pour la prévision du niveau de bruit hydrodynamique généré par une pompe centrifuge

Prediction of hydrodynamic noise levels, generated by blade pumps using principal energetic parameters

E. AFONIN, K. NOVOZHILOV

\section{Thème 4 / Topic 4}

Mesure du bruit hydraulique dans les pompes Measurement of fluid borne noise in pumps

Mesure des caractéristiques d'une pompe centrifuge considérée comme une source de fluctuations de pression

Measurement of the characteristics of a centrifugal pump as a source of pressure pulsations

C.A.F de JONG, P.C. KRIESELS, J.C. BRUGGEMAN, E. VANBOKHORST

Utilisation d'une source de bruit externe pour la détermination de la source (Qs) et de l'impédance caractéristique (Zs) d'une pompe centrifuge monoétagée.

Investigation of the fluid borne noise source flow (Qs) and source impedance ( $\mathrm{Zs}$ ) characteristics of a single stage centrifugal pump using a secondary noise source

R.J. WHITSON, I.M. BENSON

\section{Thème 5 / Topic 5}

Mesure des bruits aérien et solidien

Structure-borne and air-borne noise measurement

Le bruit des pompes: contraintes et enjeux

Pump noise; constraints and challenges

J. DUMONT

Bruit et vibrations des pompes centrifuges

Noise and vibrations of centrifugal pumps

J. DE WAELE

Mesures de vibrations pour les matériels de la Marine Nationale Structureborne noise measurement techniques for french navy equipments

\section{SILHOUETTE}

Estimation du bruit par une méthode d'intensimétrie Assessment of noise levels using sound intensity measurement R. LUTY

Analyse expérimentale de problèmes vibro-acoustiques d'une pompe élémentaire

Vibro-acoustic troubleshooting solves $5 \mathrm{MW}$ boilerfeed pump testrig noise \& vibration problems

\section{GIELEN, D. VANDENBROECK}

Caractéristiques vibro-acoustiques et transmission de l'énergie dans des tuyauteries remplies de fluide

Vibro-acoustical charaeteristics and energy transmission in fluidfilled pipes

G. PAVIC, K. TRDAK, A. BADIE-CASSAGNET

\section{Thème 6 / Topic 6}

Interaction dynamique entre la pompe et le circuit Dynamic interaction between pump and system

Caractérisation acoustique d'une pompe centrifuge, modélisation dynamique et validation expérimentale

Acoustical transfer matrix for a centrifugal pump experimental validation of a matrix theory

J. TAVERNIER, M. MAGNANI, H. GAGLIARDINI, V. LAGARRIGUE, R. PERRET

Modélisation de la transmission de perturbations hydroacoustiques à travers une pompe centrifuge

Modelling transmission of hydro-acoustical disturbances through a centrifugal pump

T. JACOB, J.-E. PRENAT

Mesure de l'impédance dynamique d'une pompe en vue de l'analyse du comportement dynamique du réseau de tuyauteries associé The measurement of pump dynamic impedance for use in piping system dynamic analysis

B. FRANCE, D.W. BILYK

Génération et propagation du bruit hydraulique dans les pompes centrifuges

Generation and propagation of hydraulic noise in centrifugal pumps H. ZOGG, U. BOLLETER

Etude expérimentale et numérique des résonances acoustiques d'une pompe centrifuge

Computational and experimental study of acoustical resonances in centrifugal pump working cavity

\section{S.F. TIMUSHEV, A.K. NEDASHKOVSKY}

Oscillations à basse fréquence d'un circuit hydraulique alimenté par une pompe centrifuge

Low-frequency oscillations of hydraulic systems with centrifugal pumps

W, KOLARCIK, R, MATULA, I STRASKRABA, V LOVICAR

Interaction dynamique entre une pompe primaire et le circuit associé de la centrale nucléaire de Darlington

Acoustical characterization of the heat transport system of Darlington nuclear generation station: analysis technique and results

G. RZENTKOWSKI, J.W. FOREST, D.G. MARTIN

Analyse expérimentale des pulsations de pression dans un modèle de pompe turbine réversible

Experimental analysis of pressure pulsations in a reversible pump turbine and self excited pressure pulsations from a leaking check valve

H. BREKKE, J. JERNSLETTEN, B. SVINGEN

\section{Thème 7 / Topic 7}

Codes de calcul pour l'analyse du bruit et des vibrations

Computer codes for noise vibrations analysis

LACTUS : Logiciel d'Aide à la Conception de réseaux de TUyauteries Silencieux

LACTUS: Software for computer-aided design of silent piping systems

E. LANDEL, P. BLANC, F. DI COSTANZO

Utilisation du code PULSIM pour simuler dans un circuit l'influence des fluctuations de pression générées par une pompe centrifuge.

The simulation of pulsating flow generated by centrifugal pumps by means of the simulation program PULSIM

E. VAN BOKHORST, H. KORST, J.P.M. SMEULERS, J.C BRUGGEMAN 
CIRCUS : Code de calcul des vibrations de tuyauteries induites par les écoulements

CIRCUS : Code for calculation of flow-induced vibrations in piping system

D. de MONTUSSAINT, J.L. TROLLE, V. VILLOUVIER

Etude paramétrique des oscillations non périodiques d'un réseau de tuyauteries parcouru par un fluide

Nonperiodic motions in pipes conveying fluid; a parametric study

C. FRANCIOSI, M.A. DE ROSA

\section{Thème 8 / Topic 8}

Méthodes expérimentales d'analyse du bruit et des vibrations des pompes et des circuits

Experimental methods for noise and vibration analysis of pumps and piping systems

Identification des coefficients de force et de moment de roues de pompe alimentaire

Identification of force and moment coefficients on full scale boilerfeed pump impellers

J. VERHOEVEN, D. DE VIS, L. GIELEN

Vibrations sous-synchrones de pompes centrifuges multi-étagées fonctionnant à débit partiel

Subsynchronous vibrations in multistage centrifugal pumps running at part capacity

B. MATZ, D.H. HELLMANN

Analyse expérimentale des sollicitations dynamiques appliquées à un réseau de tuyauteries

Experimental analysis of dynamic loads applied on a piping system J.L. TROLle, P. VAUGRANTE, S. FRIKHA, G. COFFIGNAL

Méthodes de diagnostic des problèmes de vibration de pompes Systematic diagnosis of pump vibration problems

E. EGUSQUIZA, M.F. WHITE

Analyse de fluctuations de pressions en conduite

Pressure fluctuations analysis in piping system

J. CHARLEY, A. HAOUAS

Influence de la ligne de mesures sur l'estimation des fluctuations de pression générées par une pompe

Estimation of pump-generated pressure pulsations from instrument line measurements

\section{G. RZENTKOWSKI, J.W. FOREST, J.H. RUSSEL}

Les possibilités de l'interférométrie holographique pour l'étude des vibrations d'une roue en rotation

Possibilities of holographic interferometry for studying vibrations of a rotating impeller

\section{A. BONAZZI, V. LAGARRIGUE, R. PERRET}

\section{Thème 9 / Topic 9}

Etude de cas industriels

Case histories

Analyse numérique et expérimentale du comportement vibratoire d'un circuit de centrale nucléaire sous l'excitation hydraulique de sa pompe de circulation

Numerical and experimental analysis of the vibratory behaviour of a nuclear power plant piping system excitated by a pump

E. VATIN, J. GUILLOU, F. TEPHANY, C. TROLLAT

Analyse des fluctuations de pression générées par les pompes du circuit primaire d'un réacteur à eau pressurisée
Pump-induced acoustical analysis of the primary coolant system in a pressurized water reactor

R.E. SCHWIRIAN

Des essais sur site pour résoudre les problèmes de vibrations de pompes

Field-tested solutions to pump vibrations

T. NAKATO, J.D. MOLITOR, R.J. SCHWEPPE

Problèmes hydroacoustiques sur un circuit de la centrale nucléaire de Darlington

Darlington fuel damage investigation

A.D. TELFER

Etude des instabilités, à deux fois la fréquence de rotation, du rotor d'une pompe centrifuge monoétagée

Rotor instability in a single-stage centrifugal pump super synchronous whirling at almost twice the operating speed: a case history JJ. VERHOEVEN, T. FENG, T. NEUMER

\section{Thème 10 / Topic 10}

Réduction du bruit et des vibrations des pompes Pump noise and vibration control

Développement, vis-à-vis des bruits et vibrations, des pompes utilisées dans le bâtiment,

Improving pumps used in building-trade in terms of noise and vibration

AMAND, BRUNET, MATTE, POTTIER

Exemple de réduction des fluctuations de pression sur une pompe centrifuge

Example of reduction in pressure fluctuations in a centrifugal pump

F. DI COSTANZO, F. ROULE, D. CARAGLio

Interaction dynamique entre une pompe et un circuit

Dynamic interaction between pump and piping system

\section{R.T. HARTLEN, J.T. URBANOWICZ, S.L. BARRECA}

Surveillance vibratoire et acoustique des pompes centrifuges

Vibration and noise monitoring of centrifugal pumps

O. PRAKASH, K. GUPTA, B. NAKRA

Phénomènes instationnaires sur les aubes de pompes axiales des propulseurs.

Les méthodes de réduction du bruit créé par la cavitation et la turbulence

Unsteady phenomena on axial pump blades. Methods for reducing noise due to cavitation and turbulence.

A.A. ROUSSETSKY

Simulation d'atténuation du bruit hydraulique des pompes centrifuges par action sur les impédances acoustiques de la pompe

A simulation study on the attenuation of fluid-borne noise of centrifugal pumps by influencing the acoustic impedances of the pump

E. KLAUI

Réduction du bruit par couplage dynamique entre une pompe et un silencieux

Using of dynamic interaction between pump and pulsation muffler for noise decreasing

\section{R. STAROBINSKY}

Méthodes de réduction du bruit des pompes

Noise réduction methods in machines hydraulic actuators

Y.V. CHUPRYNIN, A.S. SHAGINYAN

Conception du rotor des pompes régénératives

Impeller design for side channel flow machines

D. SUREK 